\title{
Neurophysiological correlates of habituation during exposure in spider phobia
}

\author{
Dick J. Veltman ${ }^{\mathrm{a}, *}$, Wim E. Tuinebreijer ${ }^{\mathrm{b}}$, Daniël Winkelman ${ }^{\mathrm{c}}$, \\ Adriaan A. Lammertsma ${ }^{c}$, Menno P. Witter ${ }^{\mathrm{d}}$, \\ Raymond J. Dolan ${ }^{\mathrm{e}}$, Paul M.G. Emmelkamp ${ }^{\mathrm{b}}$ \\ a Department of Psychiatry and Clinical PET Center, Vrije Universiteit Medical Center, De Boelelaan 1117, P.O. Box 7057, \\ 1007 MB Amsterdam, The Netherlands \\ ${ }^{\mathrm{b}}$ Department of Clinical Psychology, University of Amsterdam, Roetersstraat 15, 1018 WB Amsterdam, The Netherlands \\ ${ }^{\mathrm{c} C l i n i c a l}$ PET Center, Vrije Universiteit Medical Center, De Boelelaan 1117, P.O. Box 7057, 1007 MB Amsterdam, The Netherlands \\ ${ }^{\mathrm{d} D e p a r t m e n t ~ o f ~ A n a t o m y, ~ V r i j e ~ U n i v e r s i t e i t ~ M e d i c a l ~ C e n t e r, ~ v d ~ B o e c h o r s t s t r a a t ~ 7, ~} 1081$ BT Amsterdam, The Netherlands \\ ${ }^{\mathrm{e}}$ Wellcome Department of Cognitive Neurology, Institute of Neurology, Queen Square 12, London WC1, UK
}

Received 28 October 2003; received in revised form 23 July 2004; accepted 11 September 2004

\begin{abstract}
Imaging studies using symptom-provocation paradigms in specific phobia have yielded contradictory results, possibly reflecting a failure to account for habituation processes. Given that a single session of exposure in vivo can result in significant improvement in specific phobia, we used prolonged exposure to phobic stimuli to identify CNS regions showing habituation. Eighteen subjects (12 with spider phobia, 6 healthy controls) underwent $\mathrm{H}_{2}^{15} \mathrm{O}$-positron emission tomography while being continuously presented with pictures of spiders or butterflies. Results showed main effects (spiders>butterflies) in the phobia group in the left fusiform gyrus (FG) and the right parahippocampal gyrus (PHG), with bilateral perirhinal cortex and right limbic areas approaching significance. Group $\times$ condition effects were found in the right amygdala and PHG. During spider scans, large habituation effects were observed in the anterior medial temporal lobe (MTL) bilaterally. Regression analyses demonstrated that state anxiety was correlated with activity in left amygdala, bilateral perirhinal cortex, right FG, and periaquaductal grey; by contrast, phobic fear was only associated with right-sided hippocampal activity. We conclude that prolonged exposure to phobic stimuli is associated with a significant decrease in bilateral anterior MTL regional cerebral blood flow. Right anterior MTL, identified when comparing phobic vs. neutral stimuli and habituation to phobic vs. neutral stimuli in the phobia group, implicates this region in phobic fear. Analyses of covariance suggest a further functional segregation with state anxiety being linked to enhanced activity in amygdala,
\end{abstract}

* Corresponding author. Tel.: +3120 4449639; fax: +31 204449636.

E-mail address: dj.veltman@vumc.nl (D.J. Veltman). 
perirhinal cortex, and tegmentum, and phobic fear with enhanced right hippocampal activity, suggesting a neuroanatomical differentiation between emotional-vegetative and cognitive aspects of (phobic) fear.

(c) 2004 Elsevier Ireland Ltd. All rights reserved.

Keywords: Spider phobia; Positron emission tomography; Exposure; Habituation

\section{Introduction}

Specific phobia is a psychiatric disorder characterized by marked and persistent fear of circumscribed objects or situations, such as snakes, spiders, and being in a tunnel or elevator (American Psychiatric Association, 1994). Subjects with a specific phobia readily concede that their fears are exaggerated, but they nevertheless go to great lengths to avoid phobic stimuli. The etiology of specific phobias is unknown, although both constitutional and environmental factors may contribute to their pathogenesis (Fyer, 1998). Subtypes of specific phobias (animal, blood/injury, and situational phobias) tend to cluster in families (Skre et al., 2000; Kendler et al., 2002). In addition, previous traumatic experiences and/or vicarious learning may predispose to their onset (Lichtenstein and Annas, 2000). Exposure therapy, involving exposing patients to feared situations, is the gold standard for treatment of specific phobia. This treatment is based on the idea that anxiety subsides through a process of habituation. Habituation refers to a decline in fear responses, particularly physiological responses, with repeated exposures to fear-provoking stimuli. Several studies provide supportive evidence for the role of habituation in exposure therapy, with self-reported fear and physiological arousal showing a decline across exposure sessions, consistent with habituation (Emmelkamp, 2004).

Over the past decade, brain-imaging techniques, such as positron emission tomography (PET) and single photon emission computed tomography (SPECT), have been used to investigate the neuroanatomical substrate of specific phobias, particularly animal phobias. These studies typically involve exposing subjects to phobic objects such as snakes or spiders, either pictures or live animals, during scanning. To date, these studies have yielded conflicting results. Whereas Mountz et al. (1989) in an early PET study reported negative results, subsequent studies such as those of Wik et al. (1993) found increased regional cerebral blood flow (rCBF) in secondary visual cortex but decreased $\mathrm{rCBF}$ in hippocampus and orbitofrontal, prefrontal, temporopolar, and posterior cingulate cortex with exposure to phobic stimuli. In a follow-up study (Fredrikson et al., 1995), intravenous diazepam did not effect rCBF changes or influence subjective/physiological fear indices. Johanson et al. (1998), using ${ }^{133}$ Xe-SPECT, reported decreased right lateral prefrontal flow during presentation of a spider video compared with a neutral video, particularly in near-panicking subjects. In contrast, Rauch et al. (1995), in their subjects with small animal phobia, found increased rCBF in left posterior orbitofrontal, left insular, and left somatosensory cortex, as well as in right anterior temporal and anterior cingulate cortex, but not in the amygdala complex during presentation of phobic objects compared with baseline. Similar findings were reported by Reiman (1997).

Inconsistencies in previous findings may reflect methodological differences such as imaging modality, stimulus paradigms (eyes open or closed, visual stimuli vs. touch), and data-analytic techniques (region of interest vs. voxel by voxel methods). In addition, few studies have controlled for decrements in subjects' responses in time, due to habituation after repeated exposure to phobic stimuli. Reiman (1997) attempted to reduce habituation effects by placing the snake closer to the subject with successive scans. To our knowledge, only a preliminary report by Drevets et al. (1995) addressed the effects of repeated stimulation (using 'live' animals) on patterns of CNS activation in subjects with small animal phobia, where increased activity during habituation in left posterior orbitofrontal cortex alone was found. In normal volunteers, several studies have shown that responses to fearful stimuli, particularly in anterior medial temporal cortex, may decrease rapidly over time (Breiter et al., 1996; Buchel et al., 1999; Thomas 
et al., 2001; Wright et al., 2001). This finding has been put forward as an explanation for the failure to demonstrate increased activity in the amygdala complex in the above-reviewed studies on specific phobias (Fischer et al., 2000), in spite of the hypothesized key role of this region in fear responses (LeDoux, 1998).

Two issues are, however, important when using functional neuroimaging as a tool to investigate habituation processes. First, habituation of responses in phobia patients during repeated stimulation, even in the anterior medial temporal lobe, may reflect not only diminished fear of phobic objects, but also decreased novelty effects or anticipatory anxiety (e.g., Boshuisen et al., 2002). In the latter case, habituation effects for fear-associated stimuli would be similar to those for neutral stimuli, as reported by Fischer et al. (2000). Second, although exposure is the preferred treatment for phobia, and significant improvement may occur after a single session (Ost, 1989), responses to phobic stimuli may be more resistant to extinction than those to fearful stimuli in normal volunteers. Therefore, brief presentations (1-2 min for each scan, as is customary for ${ }^{15} \mathrm{O}$-PET) may not be sufficient to achieve a reduction of phobic fear.

In the present study, $\mathrm{H}_{2}^{15} \mathrm{O}$-PET was used to investigate the neurophysiological correlates of habituation during prolonged exposure in spider phobia patients. To this end, visual stimuli were presented not only during each scanning period, but continuously in order to mimic an exposure therapy session as closely as possible. In addition, ratings of both actual distress and phobic fear were obtained with the aim of differentiating between non-specific, fluctuating state anxiety and specific, enduring phobic fear. Based on results of previous functional magnetic resonance imaging (fMRI) studies in normal volunteers, it was expected that habituation would be associated with decreased regional blood flow in bilateral anterior medial temporal lobe (MTL), as would also be predicted by the model proposed by LeDoux (1998).

\section{Methods}

\subsection{Subjects}

Eighteen subjects (12 spider phobia patients and 6 control subjects) participated in the study. Twelve subjects (11 females, 1 male, mean age $=32.1 \pm$ S.D. 14.0 years) were diagnosed as spider phobics based on their scores on the Fear of Spiders Questionnaire (FSQ) and the Spider Phobia Questionnaire (SPQ) (Muris and Merckelbach, 1996). Scores on the FSQ and SPQ for phobic subjects were $77.4 \pm 21.4$ and $19.5 \pm 9.5$, respectively, in agreement with scores reported by Muris and Merckelbach (89.1 \pm 19.6 and $23.2 \pm 2.9$ for their phobic subjects). Six age-matched control subjects were also included (4 females, 2 males; mean age $=30.5 \pm$ S.D., 10.6 years; FSQ score $=7.2 \pm 10.9$, SPQ score $=2.8 \pm 3.8$ ). Subjects with neurological and psychiatric disorders were excluded. In addition, pregnancy, inadequate contraception, and use of psychoactive substances were exclusion criteria. The study was approved by the Ethics Committee of the Vrije Universiteit Medical Center. Informed consent was obtained from all participants.

\subsection{Exposure paradigm}

Each subject was presented with nine 9.5-min series of 95 pictures of either spiders or butterflies, each of which was shown for $6 \mathrm{~s}$ without a break. A session contained six series of spider pictures and three series of butterfly pictures, suitably randomised across subjects. Butterflies were included to control for non-specific habituation effects, as they are visually similar to spiders. During each series, a 90-s PET scan was performed after 5-6 min of exposure. Stimuli were drawn from two sets (spiders and butterflies) of approximately 200 colour pictures each. Subjects therefore saw each picture two to four times. Care was taken, however, that only new pictures were presented during each scan. Using a personal computer, pictures were presented on a flat screen positioned in front of each subject, at a distance of about $0.5 \mathrm{~m}$ from the eyes. Subjects were instructed to concentrate on the task and watch each item carefully. After each series of pictures, subjects were asked to rate subjective distress (Subjective Units of Distress Scale; SUD-S) and fear for spiders on a 100-point scale. Blood pressure (RR) and heart rate (HR) were automatically recorded every $2.5 \mathrm{~min}$ using a cuff around the left upper arm (RR) and a sensor on the right middle or index finger (HR). 


\subsection{Scanning details}

PET scanning was performed using a Siemens ECAT EXACT HR+(CTI/Siemens, Knoxville, TN $)$ with an axial field of view of $15 \mathrm{~cm}$. Before each scanning session, a cannula was inserted into the left antecubital vein and normal saline was infused. After a transmission scan was performed, for each of the nine emission scans, $10-15 \mathrm{ml}$ normal saline containing 370-400 MBq of $\mathrm{H}_{2}^{15} \mathrm{O}$ was administered as a bolus injection using a MedRad infusion pump. Data acquisition lasted $90 \mathrm{~s}$, and the interval between successive $\mathrm{H}_{2}^{15} \mathrm{O}$ injections was 10 min to allow for radioactive decay. Image reconstruction was done using an ordered subset-expectation maximisation (OSEM) method, with four iterations and 16 subsets (Boellaard et al., 2001; Mesina et al., 2002), followed by motion-corrected attenuation correction (Boellaard et al., 2002), with the aim of increasing the signal-to-noise ratio and of controlling for motionrelated reconstruction artifacts. A T1-weighted magnetic resonance image (MRI) was acquired from each subject with a 1.5-T Sonata (Siemens, Erlangen) MR scanner [magnetisation prepared-rapid acquisition gradient echo (MP-RAGE); inversion time: $300 \mathrm{~ms}$, $\mathrm{TR}=15 \mathrm{~ms}, \mathrm{TE}=7 \mathrm{~ms}$, flip angle $=8^{\circ}$, voxel size $1 \times 1$ $\times 1.5 \mathrm{~mm}]$.

\subsection{Statistical analysis}

Psychometric and physiological data were analyzed using a standard statistical package. Imaging data were analyzed with SPM99 software (http:// www.fil.ion.ucl.ac.uk). After reconstruction, PET images were realigned, and each subject's structural MRI was coregistered to the mean PET image. Next, PET images were transformed to approximate Talairach (Talairach and Tournoux, 1988) anatomical standard space, as defined by the SPM99 T1 template, using the transformation matrix obtained from warping each subject's coregistered T1 image. PET images were resampled to $3 \times 3 \times 3 \mathrm{~mm}$ voxels and smoothed using a 10-mm full-width/half-maximum (FWHM) Gaussian filter. Following spatial preprocessing, PET data were analyzed using a linear regression model, and weighted contrasts were computed for condition and time effects for each group, and for group$\times$ condition interactions. Additional regression analy- ses were performed to assess correlations between imaging data and psychometric and physiological measurements. Results are reported at $P<0.05$, corrected for multiple comparisons using the False Detection Rate (FDR) method (Genovese et al., 2002), unless indicated otherwise.

\section{Results}

\subsection{Psychometric and physiological data}

One subject (from the phobia group) complained of sleepiness during the scanning session; these data were excluded from the analysis. In the remaining 11

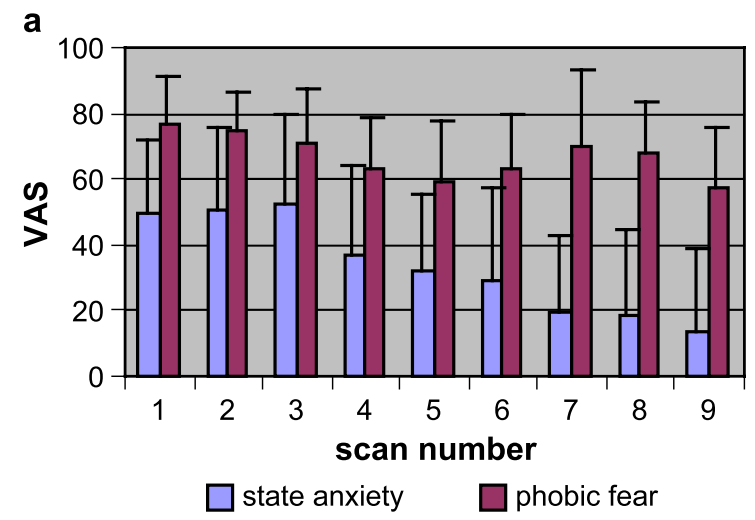

b

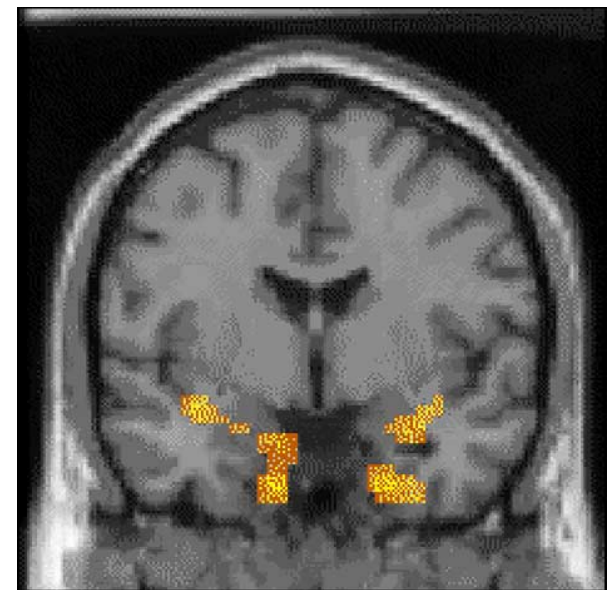

Fig. 1. (a) State anxiety (SUD-S) and phobic fear ratings during spider scans (1-6) and butterfly scans (7-9) in phobic subjects ( $n=11)$. (b) Habituation (signal decrease over time) during spider scans in the phobia group $(n=11)$. 
subjects in the phobia group, state anxiety (as measured with the SUD-S) during exposure to spider pictures decreased from $49.6 \pm 22.4$ to $29.6 \pm 22.7$ (Fig. 1a). Paired comparisons showed that the main decrease in SUD-S occurred between the third and fourth spider scan $(t=4.6, P<0.001)$. Fear of spiders decreased from $76.4 \pm 15.2$ to $62.7 \pm 17.1$, also with the only significant change occurring between the third and fourth spider scan $(t=2.6, P<0.05)$. Surprisingly, pairs of ratings (SUD-S and fear of spiders) for each scan did not show statistically significant correlations, with $r$ 's ranging between -0.18 and +0.38 .

Mean state anxiety in the phobia group was higher than in controls during the spider scans $(41.9 \pm 23.9$ vs. 13.6 $\pm 9.1, P<0.01)$ but not during butterfly scans $(17.0 \pm 24.5$ vs. $13.5 \pm 9.3$, n.s.). In contrast, fear of spiders was higher in the phobia group both during spider scans $(68.1 \pm 13.9$ vs. $15.4 \pm 10.4, P<0.0001)$ and butterfly scans $(65.4 \pm 17.7$ vs. $16 \pm 10.8$, $P<0.0001)$. In the control group, neither the decrease in SUD scores $(15.8 \pm 9.1$ to $12.5 \pm 9.6)$ nor the decrease in ratings of fear of spiders $(18.7 \pm 11.1$ to $13.3 \pm 10.8$ ) was statistically significant.

Mean arterial blood pressure in the two groups was similar during spider scans $(86.3 \pm 7.0$ vs. $86.6 \pm 6.9$, n.s) and control scans $(86.0 \pm 6.4$ vs. $86.3 \pm 6.2$, n.s $)$. Differences in heart rate between the two groups were also not observed (spider scans: $65.0 \pm 6.8$ vs.

Table 1

Main effects (phobia group, $N=11$ ) for spider pictures vs. butterfly pictures

\begin{tabular}{lrrlll}
\hline Spiders $>$ butterflies & & & & & \\
\hline Region & Coordinates & & BA & $Z$-score \\
\hline R lateral prefrontal cortex & 51 & 9 & 27 & 44 & $3.5^{*}$ \\
R posterior insula & 39 & -3 & -3 & & $3.5^{*}$ \\
L temporal & & & & & \\
$\quad$ Perirhinal cortex & -33 & -12 & -39 & $38 / 20$ & $3.8^{*}$ \\
& -24 & -6 & -45 & $38 / 20$ & $3.4^{*}$ \\
R temporal & & & & & \\
Amygdala & 15 & 0 & -21 & & $3.3^{*}$ \\
Perirhinal cortex & 33 & -6 & -42 & $38 / 20$ & $3.8^{*}$ \\
$\quad$ Parahippocampal gyrus & 36 & -33 & -24 & 36 & 4.2 \\
L fusiform gyrus & -36 & -42 & -24 & 20 & 4.6 \\
& -45 & -69 & -9 & 37 & 4.2 \\
Thalamus & 18 & -21 & -6 & & $3.6^{*}$ \\
\hline
\end{tabular}

$\mathrm{BA}=$ Brodmann area.

* $P<0.1$ corrected for multiple comparisons.
Table 2

Condition (spider vs. butterfly pictures) by group [spider phobia $(N=11)$ vs. controls $(N=6)]$ interaction effects

\begin{tabular}{lrrrrr}
\hline Condition $\times$ group effects & \multicolumn{7}{l}{} \\
\hline Region & Coordinates & & BA & $Z$-score \\
\hline Right temporal & & & & & \\
Amygdala & 15 & 3 & -18 & - & 3.4 \\
Parahippocampal gyrus & 33 & -30 & -21 & 36 & 3.3 \\
\hline
\end{tabular}

$\mathrm{BA}=$ Brodmann area.

$60.3 \pm 6.4$, n.s.; butterfly scans: $63.0 \pm 4.8$ vs. $59.8 \pm 6.4$, n.s.).

\subsection{Imaging data}

\subsubsection{Main effects: spiders vs. butterflies}

Table 1 presents the main effects for pictures of spiders (compared with butterflies) in the phobia group. At the chosen threshold $(P<0.05$, FDRcorrected, which was equivalent to $Z>4.0$ for this contrast), activations were found in left fusiform and right parahippocampal gyrus. At a slightly lower threshold of $P<0.1$ corrected $(Z>3.4)$, we found activations in right lateral prefrontal cortex, bilateral perirhinal cortex, right pulvinar, right posterior insula, and right medial amygdalar region.

The reverse contrast did not show significant activations, nor did we observe significant main effects in the control group. Condition (spiders vs. butterflies $) \times$ group interactions showed specific effects in the phobia group in right amygdala and right parahippocampal gyrus (Table 2).

Table 3

Habituation effects (phobia group, $N=11$ ) for spider pictures

\begin{tabular}{lrrrrl}
\hline Habituation: spiders & \multicolumn{3}{l}{ B-score } \\
\hline Region & \multicolumn{2}{l}{ Coordinates } & & BA & Z \\
\hline Left temporal & & & & & \\
$\quad$ Anterior inferior & -24 & 6 & -36 & 28 & 3.9 \\
Amygdala & -24 & 0 & -18 & 34 & 3.8 \\
$\quad$ Perirhinal cortex & -12 & -6 & -39 & 36 & 4.3 \\
Right temporal & & & & & \\
$\quad$ Anterior inferior & 36 & 21 & -27 & 38 & 4.8 \\
Amygdala & 24 & 0 & -21 & & 3.4 \\
$\quad$ Perirhinal gyrus & 24 & -9 & -42 & 36 & 4.1 \\
Hypothalamus & 6 & -3 & -9 & & 3.7 \\
\hline
\end{tabular}

$\mathrm{BA}=$ Brodmann area. 
Table 4

Habituation $\times$ condition effects (phobia group, $N=11$ ) for spider vs. butterfly pictures

\begin{tabular}{|c|c|c|c|c|c|}
\hline \multicolumn{6}{|c|}{ Habituation: spiders $>$ butterflies } \\
\hline Region & \multicolumn{3}{|c|}{ Coordinates } & $\mathrm{BA}$ & $Z$-score \\
\hline \multicolumn{6}{|l|}{ Prefrontal } \\
\hline Anterior cingulate & 4 & 34 & 18 & 24 & 3.7 \\
\hline \multicolumn{6}{|l|}{ Left temporal } \\
\hline Anterior inferior & -24 & 6 & -36 & 28 & 3.9 \\
\hline Amygdala & -22 & 0 & -16 & & 3.3 \\
\hline Perirhinal gyrus & -10 & -6 & -38 & 36 & 4.1 \\
\hline \multicolumn{6}{|l|}{ Right temporal } \\
\hline Anterior inferior & 38 & 20 & -24 & 38 & 4.3 \\
\hline Amygdala & & & & & n.s. \\
\hline Perirhinal gyrus & 22 & -8 & -40 & 36 & 3.6 \\
\hline Hypothalamus & 6 & -2 & -10 & - & 3.3 \\
\hline
\end{tabular}

$\mathrm{BA}=$ Brodmann area.

\subsubsection{Habituation/sensitization}

In the phobia group, as expected, large habituation effects for spider stimuli (signal decrease as a linear function of time) were found bilaterally in the anterior medial temporal lobe, including the amygdala, extending bilaterally into posterior insular cortex, and hypothalamus (Table 3, Fig. 1b). Post hoc paired comparisons revealed that right amygdala activity decreased mainly between the first and second spider scans, whereas left amygdala activity showed a significant decline between the second and third spider scans. Sensitization effects for spider stimuli (signal increases as a linear function of time) were not found.

Habituation $\times$ condition (spiders vs. butterflies) interactions in the phobia group were found in bilateral perirhinal cortex, anterior cingulate cortex, left anterior temporal pole including the amygdala region, and right anterior pole extending into posterior insula (Table 4). In the control group, we did not find significant habituation $\times$ condition interactions.

\subsubsection{Regression analyses}

Additional regression analyses were performed to identify areas in which activity correlated with physiological or behavioral data. Scan-to-scan variability in heart rate and mean arterial blood pressure were both positively correlated with activity in periaquaductal grey matter, as well as with activity adjacent to lateral temporal poles, the latter presumably due to extracranial sources (Reiman et al., 1989; Drevets et al., 1992). State anxiety, as measured with the SUD-S, was associated with enhanced activity in left amygdala and bilateral perirhinal cortex, as well as right fusiform gyrus and periaquaductal grey matter. Fear of spiders (after regressing out state anxiety) was not correlated with $\mathrm{rCBF}$ changes, although at a slightly lower threshold ( $P<0.1$, FDR corrected, or $Z>4.3$ for this contrast), a correlation with rCBF in the right hippocampus was found (Table 5).

\section{Discussion}

In the present study, we used PET to investigate neurophysiological changes (habituation) during repeated exposures to phobic stimuli in spider phobia patients. To this end, a behavioral therapy paradigm (prolonged visual stimulation) was adapted by also including a neutral control condition (pictures of butterflies). Psychometric data showed that this

Table 5

Areas showing activity associated with state anxiety (SUD-S) in fear of spiders (phobia group)

\begin{tabular}{|c|c|c|c|c|c|c|c|c|c|c|}
\hline \multirow[t]{2}{*}{ Region } & \multicolumn{5}{|c|}{ VAS state anxiety } & \multicolumn{5}{|c|}{ VAS phobic fear } \\
\hline & \multicolumn{3}{|c|}{ Coordinates } & BA & $Z$-score & \multicolumn{3}{|c|}{ Coordinates } & BA & $Z$-score \\
\hline \multicolumn{11}{|l|}{ Left temporal } \\
\hline Amygdala & -21 & 3 & -18 & & $4.0^{*}$ & & & & & \\
\hline Perirhinal gyrus & -12 & -6 & -36 & 36 & $3.8^{*}$ & & & & & \\
\hline \multicolumn{11}{|l|}{ Right temporal } \\
\hline Perirhinal gyrus & 24 & 0 & -39 & 38 & 4.6 & & & & & \\
\hline Hippocampus & & & & & & 30 & -15 & -18 & & $4.3^{*}$ \\
\hline Right fusiform gyrus & 39 & -36 & -27 & 36 & $3.7^{*}$ & & & & & \\
\hline Periaquaductal grey & -3 & -24 & -15 & & $4.3^{*}$ & & & & & \\
\hline
\end{tabular}

$\mathrm{BA}=$ Brodmann area.

* $P<0.1$ corrected for multiple comparisons. 
paradigm was successful in reducing not only state anxiety as measured with the SUD-S, but also phobic fear, albeit to a lesser extent. In the phobia group, phobic fear was similar during spider and neutral scans, as expected given that spider phobia is a trait characteristic; in contrast, state anxiety was significantly higher during the spider scans. The imaging data, on the other hand, showed a clear, if incomplete, dissociation between main effects for condition and habituation effects. Main effects for spider vs. butterfly pictures were found predominantly in fusiform/ parahippocampal gyrus, but also in bilateral perirhinal cortex, right posterior lateral prefrontal cortex, and right medial amygdalar region. Habituation effects (decreases over time) were found exclusively in socalled limbic structures involving the bilateral anterior medial temporal lobe including the amygdala, as well as in the posterior insula, and hypothalamus.

Main effects for phobic vs. neutral stimuli in the present study are only partially in agreement with those of previous imaging studies in phobic subjects. In contrast to the studies of Fredrikson et al. (1995) and Johanson et al. (1998), no significant deactivations were found. Activity in insular and posterior thalamic regions was found in accordance with observations by Reiman (1997) and Rauch et al. (1995), although their finding of medial prefrontal activity was not replicated. In a recent fMRI study by Paquette et al. (2003), spider phobia subjects were scanned twice, before and after four sessions of cognitive behavior therapy. Before therapy, viewing film excerpts depicting spiders was associated with activity in anterior right dorsolateral prefrontal cortex, as well as in visual association areas and parahippocampal gyrus. In the present study, additional main effects were identified not only in bilateral fusiform gyrus, but also perirhinal cortex, and right inferior frontal gyrus. The fusiform gyrus has been reported earlier in imaging studies using anxiogenic stimuli in normal volunteers (Breiter et al., 1996; Morris et al., 1998; Williams et al., 2001), and this may reflect emotional modulation of visual processing systems. Perirhinal cortical activity has been found to predict successful encoding (e.g., Strange et al., 2002), possibly through association of higher-order sensory inputs with emotion-related inputs from the amygdala (Kajiwara et al., 2003). This effect, we suggest, reflects an automatic engagement of mem- ory-related processes for a phobic object consequent upon emotional modulation. The right inferior frontal gyrus, on the other hand, previously implicated in declarative (fact) processing of fear (Williams et al., 2001), may reflect an obligatory retrieval of previous episodes of fear.

In the present study, extensive habituation in bilateral anterior MTL, including the amygdala, was found. This phenomenon has not previously been reported in phobia patients, but may account for the negative findings regarding the anterior MTL in earlier imaging studies, as suggested by Fischer et al. (2000). Habituation of amygdalar responses to anxiogenic stimuli (fearful faces) in normal volunteers has been described by several authors (Breiter et al., 1996; Morris et al., 1996; Fischer et al., 2000; Wright et al., 2001; Phillips et al., 2001). Most investigators have found habituation effects in the right amygdala, but not in the left, which has led to speculations that the right amygdala might be involved in rapid emotional stimulus detection, whereas the left amygdala might be associated with sustained stimulus evaluation. This hypothesis is, however, not supported by the present data. Possible explanations include differences in stimulus paradigm (spiders vs. angry faces), subject groups (phobic patients vs. normal controls) and, presumably most important, stimulus presentation duration (ca. $60 \mathrm{~min}$ vs. a maximum of $10 \mathrm{~min}$ ). This period may be critical in view of the finding that left amygdalar activity decreased, mainly between the second and third spider scans (i.e., after 15-25 min of exposure). In contrast, right amygdalar activity showed a significant decline between the first and second spider scans (after 5-15 min of exposure). This finding suggests that left amygdalar habituation might have been missed in several earlier studies because the stimulation interval might was too short. It also indicates that diminished amygdalar activity may antedate a decrease in state anxiety and/or phobic fear, which occurred mainly between the third and fourth spider scan.

In the present study, regions specific for spider phobia habituation were found in bilateral perirhinal and posterior insular cortex, as well as right hypothalamus and right amygdala, but not left amygdala. This implies that the involvement of the left amygdala, in this paradigm at least, is not primarily associated with specific fears, but may also be due 
to, e.g., anticipatory anxiety and/or novelty. This conclusion is supported by the finding that group by condition interactions specifically involved the right amygdala and parahippocampal gyrus, although these findings need to be interpreted with caution in view of the small control group. Interestingly, in the Paquette et al. (2003) study, pretreatment activity in parahippocampal gyrus disappeared after treatment, explained as due to deconditioning of contextual fear, similar to the present study.

Additional regression analyses showed that state anxiety was associated with activity in left amygdala and bilateral perirhinal cortex, as well as right fusiform gyrus, and periaquaductal grey matter, whereas phobic fear was associated with right hippocampal activity only. The latter finding again suggests that habituation to phobic stimuli in this paradigm is correlated with activity in right limbic structures. The association of amygdalar and tegmental activity with state anxiety and heart rate/arterial blood pressure is in line with earlier findings linking these structures to emotional and autonomic aspects of fear responding (Critchley et al., 2000).

Regarding the role of the hippocampus, Williams et al. (2001) reported that presentation of anxiogenic stimuli in normal volunteers led to amygdalar activation only if a concomitant change in skin conductance occurred. Anxiogenic stimuli that did not elicit skin conductance responses were associated with hippocampal activity. These findings were interpreted as implying a neuroanatomical dissociation between visceral experience and declarative fact processing of fear, as outlined by LeDoux (1998).

Although the present study was not designed to test this model, the data appear to be in agreement with such a 'dual system' hypothesis. Phobic fear was found to be correlated with right hippocampal activity only, after regressing out state anxiety, indicating that the right hippocampus is involved in cognitive aspects of (phobic) fear (Bechara et al., 1995). Moreover, exploratory analyses using regional blood flow data as covariates of interest indicated that right amygdala was functionally coupled with left amygdala, as well as bilateral perirhinal cortex, hypothalamus, and PAG, but not right hippocampus. In contrast, right hippocampus was functionally coupled with left hippocampus, right fusiform gyrus, and right posterior inferior lateral frontal cortex (data not shown).
Whereas the former regions have been implicated in both visceral and emotional aspects of fear response, the latter subsystem is similar to the hippocampallateral prefrontal network identified by Williams et al. (2001) as being involved in 'fear without arousal'.

In summary, we found that prolonged exposure to phobic stimuli during $\mathrm{H}_{2}^{15} \mathrm{O}$-PET-scanning was associated with a significant decrease in bilateral anterior medial temporal lobe (MTL) perfusion. In addition, right anterior MTL was identified when comparing phobic vs. neutral stimuli between patients and control subjects, and when comparing habituation to phobic vs. neutral stimuli in the phobia group, implicating a specific role for this region in phobic fear. Additional regression analyses suggested a further functional segregation, since state anxiety was associated with activity in amygdala, perirhinal cortex, and tegmentum, whereas phobic fear was associated with right hippocampal activity only. Given the modest size of our experimental group and the limited spatial resolution of $\mathrm{H}_{2}^{15} \mathrm{O}$-PET, these findings clearly need replication; in addition, future research should further address the issue of anterior MTL functional lateralisation, e.g., by including familiar items to account for activity related to novelty.

\section{References}

American Psychiatric Association, 1994. Diagnostic and Statistical Manual of Psychiatric Disorders, fourth edition. Author, Washington, DC.

Bechara, A., Tranel, D., Damasio, H., Adolphs, R., Rockland, C., Damasio, A.R., 1995. Double dissociation of conditioning and declarative knowledge relative to the amygdala and hippocampus in humans. Science 269 (5227), 1115-1118.

Boellaard, R., van Lingen, A., Lammertsma, A.A., 2001. Experimental and clinical evaluation of iterative reconstruction (OSEM) in dynamic PET: quantitative characteristics and effects on kinetic modeling. Journal of Nuclear Medicine 42, 808-817.

Boellaard, R., van den Heuvel, O.A., Veltman, D.J., Mesina, C.T., Lammertsma, A.A., 2002. An image registration method to correct emission-transmission scan mismatch due to task related motion in PET activation studies. Journal of Nuclear Medicine 43 (5), S362.

Boshuisen, M.L., Ter Horst, G.J., Paans, A.M., Reinders, A.A., den Boer, J.A., 2002. rCBF differences between panic disorder patients and control subjects during anticipatory anxiety and rest. Biological Psychiatry 52 (2), 126-135.

Breiter, H.C., Etcoff, N.L., Whalen, P.J., Kennedy, W.A., Rauch, S.L., Buckner, R.L., Strauss, M.M., Hyman, S.E., Rosen, B.R., 
1996. Response and habituation of the human amygdala during visual processing of facial expression. Neuron 17 (5), 875-887.

Buchel, C., Dolan, R.J., Armony, J.L., Friston, K.J., 1999. Amygdala-hippocampal involvement in human aversive trace conditioning revealed through event-related functional magnetic resonance imaging. Journal of Neuroscience 19 (24), $10869-10876$.

Critchley, H.D., Corfield, D.R., Chandler, M.P., Mathias, C.J., Dolan, R.J., 2000. Cerebral correlates of autonomic cardiovascular arousal: a functional neuroimaging investigation in humans. Journal of Physiology 523 (Pt 1), 259-270.

Drevets, W.C., Videen, T.Q., MacLeod, A.K., Haller, J.W., Raichle, M.E., 1992. PET images of blood flow changes during anxiety: correction. Science 256 (5064), 1696.

Drevets, W.C., Simpson, J.R., Raichle, M.E., 1995. Regional blood flow changes in response to phobic anxiety and habituation. Journal of Cerebral Blood Flow and Metabolism 15 (Suppl. 1), S856.

Emmelkamp, P.M.G., 2004. Behavior therapy with adults. In: Lamberts, M. (Ed.), Bergin and Garfield's Handbook of Psychotherapy and Behavior Change, 5th edition. Wiley, New York, pp. 393-446.

Fischer, H., Furmark, T., Wik, G., Fredrikson, M., 2000. Brain representation of habituation to repeated complex visual stimulation studied with PET. NeuroReport 11 (1), 123-126.

Fredrikson, M., Wik, G., Annas, P., Ericson, K., Stone-Elander, S., 1995. Functional neuroanatomy of visually elicited simple phobic fear: additional data and theoretical analysis. Psychophysiology 32 (1), 43-48.

Fyer, A.J., 1998. Current approaches to etiology and pathophysiology of specific phobia. Biological Psychiatry 44 (12), $1295-1304$.

Genovese, C.R., Lazar, N.A., Nichols, T., 2002. Thresholding of statistical maps in functional neuroimaging using the false discovery rate. Neuroimage 15 (4), 870-878.

Johanson, A., Gustafson, L., Passant, U., Risberg, J., Smith, G., Warkentin, S., Tucker, D., 1998. Brain function in spider phobia. Psychiatry Research: Neuroimaging 84 (2-3), 101-111.

Kajiwara, R., Takasima, I., Mimura, Y., Witter, M.P., Iijima, T., 2003. Amygdala input promotes the spread of excitatory neural activity from the perirhinal cortex to the entorhinal/hippocampal neurocircuit. Journal of Neurophysiology 89 (4), 2176-2184.

Kendler, K.S., Jacobson, K.C., Myers, J., Prescott, C.A., 2002. Sex differences in genetic and environmental risk factors for irrational fears and phobias. Psychological Medicine 32 (2), 209-217.

LeDoux, J.E., 1998. Fear and the brain: where have we been and where are we going? Biological Psychiatry 44 (12), 1229-1238.

Lichtenstein, P., Annas, P., 2000. Heritability and prevalence of specific fears and phobias in childhood. Journal of Child Psychology and Psychiatry 41 (7), 927-937.

Mesina, C.T., Boellaard, R., Jongbloed, G., Vaart van Der, A.W., Lammertsma, A.A., 2002. Experimental evaluation of filtered back projection versus iterative reconstruction using statistical parametric mapping analysis of PET activation studies. Journal of Nuclear Medicine 43 (5), S892.
Morris, J.S., Frith, C.D., Perrett, D.I., Rowland, D., Young, A.W., Calder, A.J., Dolan, R.J., 1996. A differential neural response in the human amygdala to fearful and happy facial expressions. Nature 383 (6603), 812-815.

Morris, J.S., Friston, K.J., Buchel, C., Frith, C.D., Young, A.W., Calder, A.J., Dolan, R.J., 1998. A neuromodulatory role for the human amygdala in processing emotional facial expressions. Brain 121 (Pt 1), 47-57.

Mountz, J.M., Modell, J.G., Wilson, M.W., Curtis, G.C., Lee, M.A., Schmaltz, S., Kuhl, D.E., 1989. Positron emission tomographic evaluation of cerebral blood flow during state anxiety in simple phobia. Archives of General Psychiatry 46 (6), 501-504.

Muris, P., Merckelbach, H., 1996. A comparison of two spider fear questionnaires. Journal of Behavior Therapy and Experimental Psychiatry 27 (3), 241-244.

Ost, L.G., 1989. One-session treatment for specific phobias. Behaviour Research and Therapy 27 (1), 1-7.

Paquette, V., Levesque, J., Mensour, B., Leroux, J.-M., Beaudoin, G., Bourguoin, P., Beauregard, M., 2003. 'Change the mind and you change the brain': effects of cognitive-behavioral therapy on the neural correlates of spider phobia. Neuroimage $18,401-409$.

Phillips, M.L., Medford, N., Young, A.W., Williams, L., Williams, S.C., Bullmore, E.T., Gray, J.A., Brammer, M.J., 2001. Time courses of left and right amygdalar responses to fearful facial expressions. Human Brain Mapping 12 (4), 193-202.

Rauch, S.L., Savage, C.R., Alpert, N.M., Miguel, E.C., Baer, L., Breiter, H.C., Fischman, A.J., Manzo, P.A., Moretti, C., Jenike, M.A., 1995. A positron emission tomographic study of simple phobic symptom provocation. Archives of General Psychiatry 52 (1), 20-28.

Reiman, E.M., 1997. The application of positron emission tomography to the study of normal and pathologic emotions. Journal of Clinical Psychiatry 58 (Suppl. 16), 4-12.

Reiman, E.M., Raichle, M.E., Robins, E., Mintun, M.A., Fusselman, M.J., Fox, P.T., Price, J.L., Hackman, K.A., 1989. Neuroanatomical correlates of a lactate-induced anxiety attack. Archives of General Psychiatry 46 (6), 493-500.

Skre, I., Onstad, S., Torgersen, S., Philos, D.R., Lygren, S., Kringlen, E., 2000. The heritability of common phobic fear: a twin study of a clinical sample. Journal of Anxiety Disorders 14 (6), 549-562.

Strange, B.A., Otten, L., Josephs, O., Friston, K., Rugg, M., Dolan, R., 2002. Dissociable human perirhinal, hippocampal, and parahippocampal roles during verbal encoding. Journal of Neuroscience 22, 523-528.

Talairach, J., Tournoux, P., 1988. A Co-planar Stereotactic Atlas of the Human Brain. Thieme, Stuttgart.

Thomas, K.M., Drevets, W.C., Dahl, R.E., Ryan, N.D., Birmaher, B., Eccard, C.H., Axelson, D., Whalen, P.J., Casey, B.J., 2001. Amygdala response to fearful faces in anxious and depressed children. Archives of General Psychiatry 58 (11), 1057-1063.

Wik, G., Fredrikson, M., Ericson, K., Eriksson, L., Stone-Elander, S., Greitz, T., 1993. A functional cerebral response to frightening visual stimulation. Psychiatry Research: Neuroimaging 50 (1), 15-24. 
Williams, L.M., Phillips, M.L., Brammer, M.J., Skerrett, D., Lagopoulos, J., Rennie, C., Bahramali, H., Olivieri, G., David, A.S., Peduto, A., Gordon, E., 2001. Arousal dissociates amygdala and hippocampal fear responses: evidence from simultaneous fMRI and skin conductance recording. Neuroimage 14 (5), 1070-1079.
Wright, C.I., Fischer, H., Whalen, P.J., McInerney, S.C., Shin, L.M., Rauch, S.L., 2001. Differential prefrontal cortex and amygdala habituation to repeatedly presented emotional stimuli. NeuroReport 12 (2), 379-383. 